\title{
Upregulated Neuro-oncological Ventral Antigen 1 (NOVA1) Expression Is Specific to Mature and Immature T- and NK-Cell Lymphomas
}

\author{
Eun Kyung Kim ${ }^{1}$ · Sun Och Yoon ${ }^{1}$ \\ Soo Hee Kim ${ }^{1,2}$. Woo Ick Yang ${ }^{1}$ \\ Yoon $\mathrm{Ah} \mathrm{Cho}^{1}$. Soo Jeong $\mathrm{Kim}^{3}$ \\ ${ }^{1}$ Department of Pathology, Yonsei University \\ College of Medicine, Seoul; ${ }^{2}$ Anatomic Pathology \\ Reference Lab, Seegene Medical Foundation, \\ Seoul; ${ }^{3}$ Department of Internal Medicine, \\ Division of Hematology, Yonsei University College \\ of Medicine, Seoul, Korea \\ Received: January 12, 2016 \\ Revised: February 5, 2016 \\ Accepted: February 8, 2016 \\ Corresponding Author \\ Sun Och Yoon, MD, PhD \\ Department of Pathology, Yonsei University College \\ of Medicine, 50-1 Yonsei-ro, Seodaemun-gu, Seoul \\ 03722, Korea \\ Tel: +82-2-2228-1763 \\ Fax: +82-2-362-0860 \\ E-mail: soyoon@yuhs.ac
}

\begin{abstract}
Background: Recent studies have revealed that the splicing factor neuro-oncological ventral antigen 1 (NOVA1) is enriched in fibroblasts and accumulated T cells of tertiary lymphoid structures. In the present study, we investigated NOVA1 expression in various subtypes of mature and immature T- and natural killer (NK)-cell lymphomas as well as in various B-cell lymphoma subtypes. Methods: NOVA1 immunoexpression was evaluated in hyperplastic palatine tonsils $(n=20), T-$ and NK-cell lymphomas $(n=177)$, diffuse large B-cell lymphomas $(n=151)$, and other types of $B$ cell lymphomas $(n=31)$. Nuclear staining intensity and percentage of positive tumor cells were graded. NOVA1 mRNA expression was analyzed in various lymphoma cell lines. Results: Tumor cells of T- and NK-cell lymphomas showed higher expression levels of NOVA1 than did normal paracortical T cells, and $56.5 \%$ of T- and NK-cell lymphoma cases showed diffuse and strong expression. The NOVA1 expression level varied according to the subtype; it was higher in angioimmunoblastic T-cell lymphoma, anaplastic lymphoma kinase (ALK)-negative anaplastic large cell lymphoma (ALCL), and T lymphoblastic leukemia/lymphoma (T-LBL), but it was lower in ALKpositive ALCL. In almost all B-cell lymphomas, NOVA1 expression was very low or negative. NOVA1 mRNA was also expressed in Jurkat, a T-LBL cell line. Conclusions: The present findings suggest that NOVA1 upregulation may be involved in certain subtypes of T- and NK-cell lymphomas, but not in B-cell lymphomas. Upregulated NOVA1 expression seems to be a specific biological feature of activated T cells such as T- and NK-cell lymphomas.
\end{abstract}

Key Words: NOVA1; Lymphoma, T-cell; NK cell lymphoma; Splicing factor
Neuro-oncological ventral antigen 1 (NOVA1) is known to play a role in the neuronal splicing program. ${ }^{1-4}$ A recent study revealed that the splicing programs of epithelial cells, fibroblasts, and endothelial cells are specifically regulated by the epithelial splicing regulatory protein 1 (ESRP1) and ESRP2, muscle blind-like splicing regulator 1 (MBNL1), NOVA1, and polypyrimidine tract binding protein 1 (PTBP1). Furthermore, RNA binding protein, fox-1 homolog 2 (RBFOX2) splicing factors, and NOVA1 are found enriched in fibroblasts. ${ }^{5}$ In our recent study, ${ }^{6}$ we also confirmed that NOVA1 is highly expressed in fibroblasts/stromal spindle cells in normal tissues.

In addition, we observed that NOVA1 is frequently upregulated in accumulated $\mathrm{T}$ lymphocytes in tertiary lymphoid structures formed by chronic inflammation, whereas only small numbers of $\mathrm{T}$ lymphocytes express NOVA1 in the secondary lymphoid organs, such as hyperplastic palatine tonsils. ${ }^{6-8}$ These findings suggest that NOVA1 upregulation may be involved in T cell activation and proliferation. ${ }^{6}$ Given that fibroblasts/stromal spin- dle cells actively regulate immune cells within the tissue microenvironment through direct interaction with immune $\mathrm{B}$ cells or T cells, ${ }^{9,10}$ NOVA1-related regulation in stromal fibroblasts/spindle cells may also occur in immune cells.

We hypothesized that NOVA1 upregulation could be involved in the pathogenesis of malignant lymphoma, which is a neoplasm of activated or transformed immune cells. In our previous study, peripheral T-cell lymphoma cases frequently showed strong NOVA1 expression while mature B-cell lymphoma cases did not, ${ }^{6}$ although the investigation was done on a small scale. In the present study, we extended our study to confirm the role of NOVA1 in the pathogenesis of malignant lymphoma. We thoroughly investigated NOVA1 expression in a large number of various subtypes of mature and immature T- and natural killer (T/NK)-cell lymphomas, and the expression of NOVA1 was also compared in various subtypes of B-cell lymphomas. 


\section{MATERIALS AND METHODS}

\section{Patients and clinical data}

Archival tissues of formalin-fixed, paraffin-embedded (FFPE) tumor blocks were retrieved from consecutively archived specimens. A set of 177 T/NK-cell lymphomas diagnosed in Severance Hospital from 1999 to 2013 were included. The subtypes of T/NK-cell lymphomas were as follows: extranodal NK/T-cell lymphoma, nasal type (NKTL; $\mathrm{n}=60$ ), peripheral T-cell lymphoma, not otherwise specified (PTCL-NOS; $\mathrm{n}=44$ ), angioimmunoblastic T-cell lymphoma (AITL; $n=16$ ), anaplastic large cell lymphoma (ALCL; anaplastic lymphoma kinase [ALK]positive, $n=16$; ALK-negative, $n=17$ ), T lymphoblastic leukemia/lymphoma (T-LBL; $n=20)$ and other types $(n=4$; hepatosplenic T-cell lymphoma, $\mathrm{n}=2$; subcutaneous panniculitis-like T-cell lymphoma, $\mathrm{n}=1$; cutaneous gamma-delta T-cell lymphoma, $n=1$ ). Clinical information was obtained from patient medical records and 139 had clinical data available. The clinicopathological characteristics are summarized in Appendix 1.

A set of 151 diffuse large $B$ cell lymphoma (DLBCL) and other types of 31 B-cell lymphomas, which were diagnosed in Severance Hospital from 2005 to 2011, were included. Other types of B-cell lymphomas were as follows: follicular lymphoma $(\mathrm{n}=14)$, mantle cell lymphoma $(\mathrm{n}=5)$, marginal zone B-cell lymphoma $(\mathrm{n}=7)$, and Burkitt lymphoma $(\mathrm{n}=5)$. Hyperplastic palatine tonsil tissues of cancer-free individuals $(\mathrm{n}=20)$ were randomly retrieved. All tumor samples were reviewed by hematopathologists based on the current World Health Organization (WHO) criteria. ${ }^{11}$ The institutional review board approved this study.

\section{Tissue microarray preparation and immunohistochemistry}

Sections of FFPE tissues were prepared and stained with hematoxylin and eosin. Under the microscope, the representative area was confirmed and selected for a 3-mm-core sized tissue microarray (TMA). One or two different representative areas per case were selected and 29 cores were embedded in a TMA block.

Immunohistochemistry of NOVA1 (1:500, Abcam, Cambridge, UK) was performed on 4- $\mu \mathrm{m}$ tissue sections using the Ventana BenchMark XT Autostainer (Ventana Medical Systems, Tucson, AZ, USA). Immunohistochemistry with other primary antibodies was performed for diagnostic purposes and the tested antibodies are listed in Appendix 2.

\section{Analysis of immunohistochemistry}

NOVA1 expression was semiquantitatively determined according to the H-score method with a total score range of $0-300 .^{12}$
The dominant intensity score of nuclear staining ( 0 , absent; 1 , weak or barely detectable; 2 , distinct brown; and 3, strong dark brown) was multiplied by the percentage of positive tumor cell nuclei. The NOVA1 protein expression score ranged from 0 to 300 , as high $(200<\mathrm{H}$-score $\leq 300)$, intermediate $(100<\mathrm{H}$-score $\leq 200)$, or low $(0 \leq \mathrm{H}$-score $\leq 100)$ expression.

\section{Cell lines and reagents}

Jurkat, a T-LBL cell line, was maintained in RPMI1640 medium (22400-089, Gibco, Life Technologies, Carlsbad, CA, USA) supplemented with $10 \%$ heat-inactivated fetal bovine serum (FBS; 6000-044, Gibco, Life Technologies). HH, a cell line derived from $\mathrm{CD} 30$-positive cutaneous T-cell lymphoma, and MAC1, a cell line derived from CD30-positive ALK-negative ALCL, were maintained in RPMI1640 medium supplemented with 20\% heat-inactivated FBS. SNK6, derived from EpsteinBarr virus (EBV)-positive NKTL, was cultured in RPMI1640 medium supplemented with $10 \%$ heat-inactivated human plas$\mathrm{ma}$ and $700 \mathrm{U} / \mathrm{mL}$ of recombinant interleukin 2. YT, an EBVpositive human NK-like leukemic cell line, was cultured in Iscove's modified Dulbecco's medium (12440-046, Gibco) supplemented with $20 \%$ heat-inactivated FBS. All the cell lines derived from T- or NK-cell lymphomas were kindly provided by Prof. Jeon YK (Seoul National University College of Medicine, Seoul, Korea). Toledo, a DLBCL cell line, was purchased from the American Type Culture Collection (ATCC, Manassas, VA, USA) and maintained in RPMI1640 supplemented with 10\% heat-inactivated FBS.

\section{RNA isolation and quantitative polymerase chain reaction}

RNA isolation was performed using an RNeasy plus mini kit (74134, Qiagen, Hilden, Germany), and cDNA was constructed using a cDNA synthesis kit (11754-050, Invitrogen, Carlsbad, CA, USA). The TaqMan probes used were as follows: GAPDH (Hs99999905-m1, AB, Life Technologies) and NOVA1 (Hs00359592-m1, AB, Life Technologies). Quantitative polymerase chain reaction was performed using $\mathrm{ABI}$ StepOnePlus under the following conditions: $50^{\circ} \mathrm{C}$ for 2 minutes, $95^{\circ} \mathrm{C}$ for 5 minutes, 40 cycles of $95^{\circ} \mathrm{C}$ for 15 seconds, and $60^{\circ} \mathrm{C}$ for minutes. Relative mRNA expression levels of NOVA1 were determined by the comparative method $\left(2^{-\mathrm{ddC}_{\mathrm{t}}}\right)$ against the reference GAPDH.

\section{Statistical analysis}

The Kruskal-Wallis (K-W), Mann-Whitney U (M-W), $t$ and $\chi^{2}$ tests were used to analyze differences between the variables ex- 
amined. Survival rates were analyzed with the Kaplan-Meier method, and differences were compared using the log-rank test. Two-sided p-values less than .05 were considered statistically significant. Statistical analyses were done using the IBM SPSS ver. 22 (IBM Corp., Armonk, NY, USA).

\section{RESULTS}

\section{Expression of NOVA1 in the palatine tonsils}

The NOVA1 expression patterns in the cell nuclei were similar to those reported in a previous study. ${ }^{6}$ Stromal spindle cells/ fibroblasts showed strong and constant expression of NOVA1. Endothelial cells variably expressed NOVA1 with negative to moderate intensity. Almost all B cells of the germinal centers or outside the germinal centers were negative for NOVA1. As for T cells, occasional expression of NOVA1 (intensity grade 2-3 with $5 \%$ or less percentage of $\mathrm{T}$ cells) was noted in the paracortical area (Table 1).

\section{Expression of NOVA1 in T/NK-cell lymphomas}

T/NK-cell lymphomas showed low to high NOVA1 expression and 99 (56.5\%) showed high NOVA1 expression (Table 1, Fig. 1). The expression level (score) of NOVA1 was higher in tumor cells of T/NK-cell lymphomas when compared to normal $\mathrm{T}$ cells of tonsil tissues (mean score, 209 vs. 7; $\mathrm{p}<.001$; nonparametric M-W) (Fig. 2A). Within T/NK-cell lymphomas, precursor T-LBL showed a relatively higher level of NOVA1 expression than did NKTL or mature (peripheral) T-cell lymphomas, although a statistically significant difference was not noted ( $\mathrm{p}=.226$; K-W) (Fig. 2B). According to the specific subtypes of T/NK-cell lymphomas, the expression level of NOVA1 in tumor cells varied $(\mathrm{p}=.011 ; \mathrm{K}-\mathrm{W})$. AITL, ALK-negative ALCL, and T-LBL showed relatively higher NOVA1 expression levels when compared to other subtypes (Table 1, Fig. 2C). Specifically, ALK-positive ALCL revealed a lower level of NOVA1 expression when compared to ALK-negative ALCL (mean score, 156 vs. $259 ; \mathrm{p}=.027 ; \mathrm{M}-\mathrm{W}$ ) and ALK-positive ALCL showed the lowest expression level among subtypes of T/NK-cell lymphomas (Table 1, Fig. 2C). The rate of high, intermediate, or low expression of NOVA1 was also different according to subtype ( $p$ $<.001$ ); high expression was relatively more frequent in AITL, ALK-negative ALCL, and T-LBL cells, while low expression was relatively more frequent in NKTL and ALK-positive ALCL (Table 1, Fig. 2D).

\section{Expression of NOVA1 in DLBCLs}

In DLBCL, high NOVA1 expression was not noted in any cases. The tested 151 DLBCL cases revealed an expression pattern with a score less than 10. Furthermore, in the various types of other B-cell lymphomas, high NOVA1 expression was not noted (Table 1, Fig. 3).

Table 1. NOVA1 expression in several types of normal and tumor immune cells

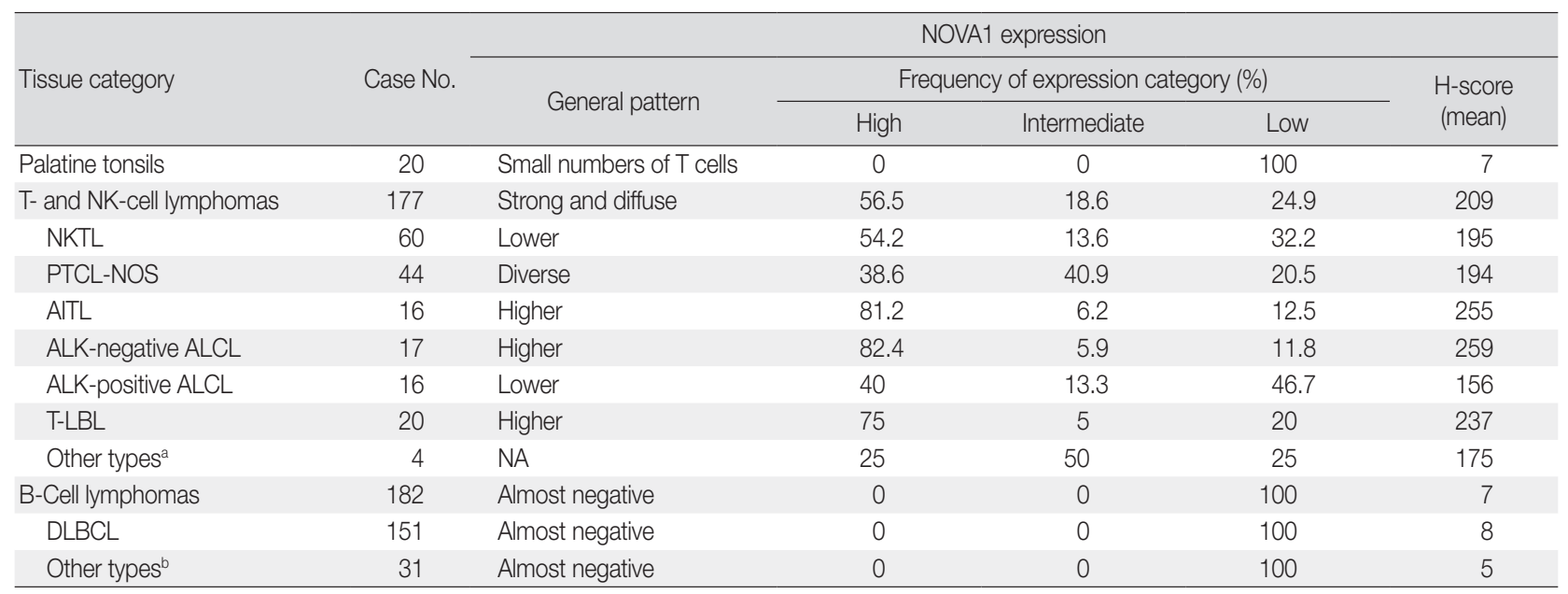

NOVA1, neuro-oncological ventral antigen 1; NK, natural killer; NKTL, extranodal natural killer/T-cell lymphoma, nasal type; PTCL-NOS, peripheral T-cell lymphoma, not otherwise specified; AITL, angioimmunoblastic T-cell lymphoma; ALK, anaplastic lymphoma kinase; ALCL, anaplastic large cell lymphoma; T-LBL, T lymphoblastic leukemia/lymphoma; DLBCL, diffuse large B-cell lymphoma.

aThe T- or NK-cell lymphomas of the "other type" category included hepatosplenic T-cell lymphoma $(n=2)$, subcutaneous panniculitis-like T-cell lymphoma $(n=$ 1), and cutaneous gamma-delta T-cell lymphoma $(n=1)$. The case number for this category was too small (only 4 cases), and therefore NOVA1 expression may not be representative for this category; "The set of "other type" B-cell lymphomas was composed of follicular lymphoma ( $n=14)$, mantle cell lymphoma ( $\mathrm{n}$ $=5)$, marginal zone B-cell lymphoma ( $n=7)$, and Burkitt lymphoma $(n=5)$. 


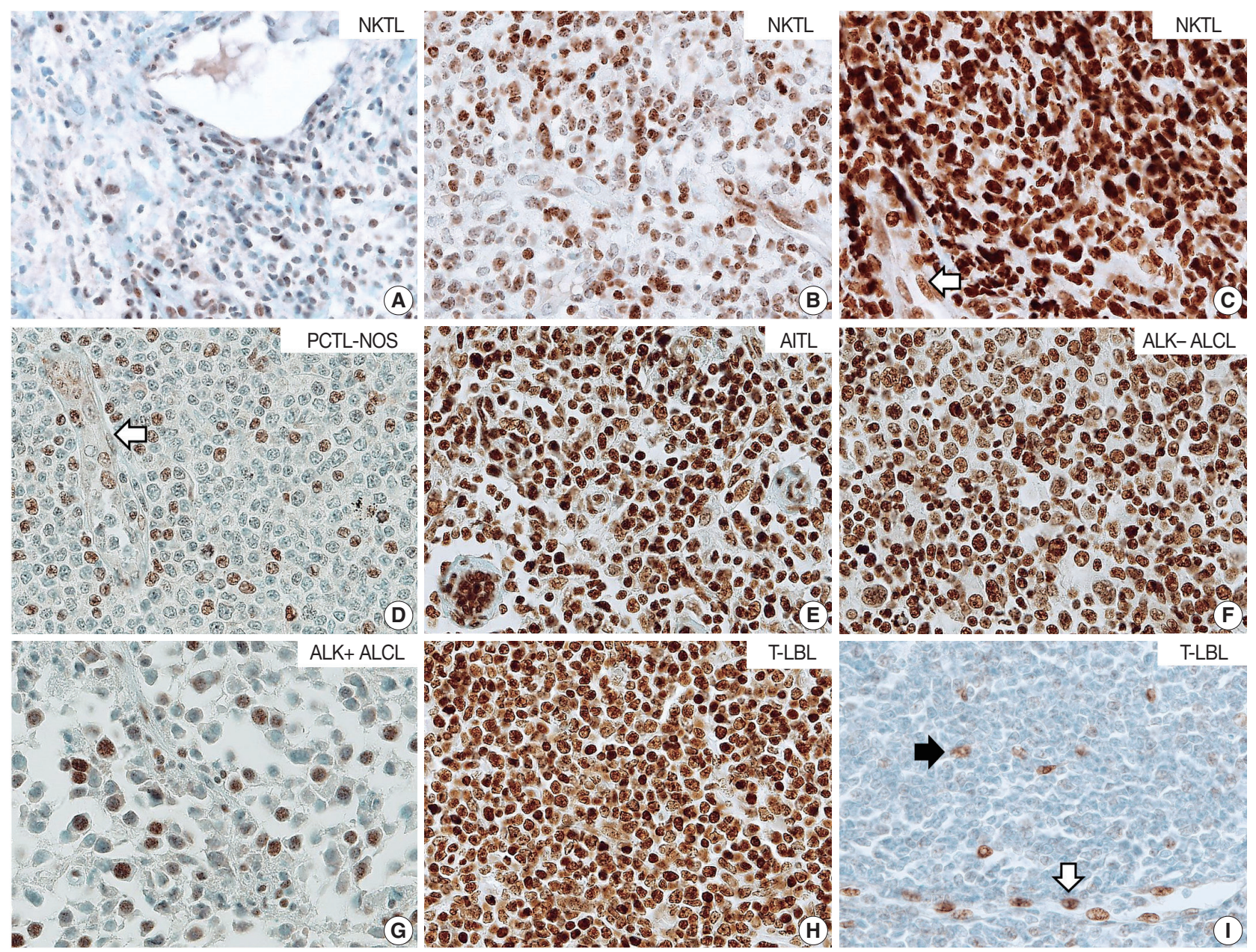

Fig. 1. Representative cases of T- and natural killer (NK)-cell lymphoma. Representative cases of extranodal NK/T-cell lymphoma, nasal type (NKTL) are presented, which show low (A), intermediate (B), and high (C) levels of neuro-oncological ventral antigen 1 (NOVA1) expression. Among mature (peripheral) T-cell lymphoma cases, low to high expression of NOVA1 is variably observed (D-G). (D) A case of peripheral T cell lymphoma, not otherwise specified (PTCL-NOS), reveals low NOVA1 expression. A case of angioimmunoblastic T-cell lymphoma (AITL) (E) and a case of anaplastic lymphoma kinase (ALK)-negative anaplastic large cell lymphoma (ALCL) (F) exhibits high NOVA1 expression. A case of ALK-positive ALCL $(G)$ demonstrates low NOVA1 expression. A case of precursor T lymphoblastic lymphoma/leukemia (T-LBL) (H) shows high NOVA1 expression while a case of T-LBL shows low NOVA1 expression. Endothelial cells exhibit high or low NOVA1 expression in accordance with the NOVA1 status of tumor T cells (white arrow in panels in C and D). In cases where most tumor cells showed negative NOVA1 expression, stromal fibroblasts (black arrow in panel I) and endothelial cells (white arrow in panel I) demonstrate positive NOVA1 expression.

NOVA1 gene expression in human lymphoma cell lines

NOVA1 mRNA was expressed only in the Jurkat cell line, which corresponds to T-LBL. NOVA1 mRNA was not detected in other tested cell lines of $\mathrm{HH}$ (correspondent to peripheral, mature T cell lymphoma), MAC1 (peripheral, mature T-cell lymphoma), SNK6 (NKTL), YT (NK cell leukemia), or Toledo (DLBCL) (Fig. 4).

NOVA1 expression and its clinicopathologic correlation in T/NK-cell lymphomas

The NOVA1 expression level in tumor cells was not different according to the organ/sites where lymphomas were growing (Appendix 3). The known clinicopathological prognostic factors such as age, lactate dehydrogenase level, Ann-Arbor stage, or International Prognostic Index (IPI) risk scores showed no correlation with NOVA1 expression. In cases of mature (peripheral) T-cell lymphomas, high expression of NOVA1 tended to be related to shorter overall survival rate in comparison to low or intermediate NOVA1 expression, although statistical significance was not observed (Fig. 5A, B). In other subtypes, such as NKTL and T-LBL, no correlation was noted between NOVA1 expression and overall survival rate. 

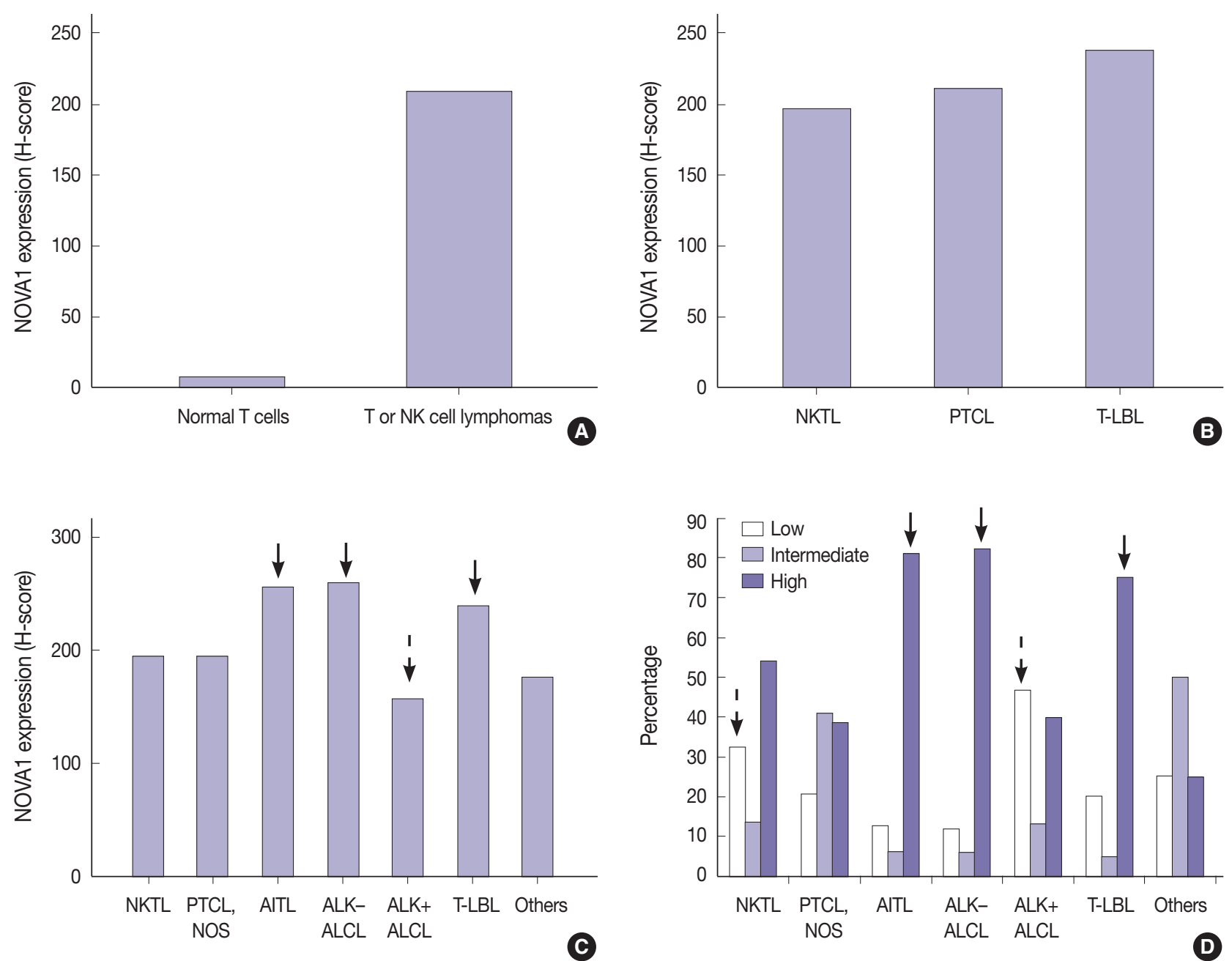

Fig. 2. Expression level of neuro-oncological ventral antigen 1 (NOVA1) in T- and natural killer (NK) cell lymphomas and normal T cells. (A) Expression level (score) of NOVA1 is higher in tumor cells of T/NK-cell lymphomas when compared to normal T cells of tonsil tissues (mean score, 7 vs 209; $\mathrm{p}<.001)$. (B) Within T/NK-cell lymphomas, T lymphoblastic leukemia/lymphoma (T-LBL) shows relatively higher levels of NOVA1 expression than do extranodal NK/T-cell lymphoma, nasal type (NKTL) or mature (peripheral) T-cell lymphomas, although a statistically significant difference is not noted $(p=.226)$. (C) According to the specific subtypes of T/NK-cell lymphomas, the expression levels of tumor cells are different $(p=.012)$. Angioimmunoblastic T-cell lymphoma (AITL), anaplastic lymphoma kinase (ALK)-negative anaplastic large cell lymphoma (ALCL), and T-LBL shows a relatively higher level of NOVA1 expression (arrow) when compared to other subtypes. ALK-positive ALCL reveals lower NOVA1 expression (dotted arrow) when compared to ALK-negative ALCL (mean score, 159 vs 256; $p=.027$ ) and ALK-positive ALCL shows the lowest expression among subtypes of T/NK cell lymphomas. (D) The rates of high, intermediate, and low expression of NOVA1 are different according to subtype $(p<.001)$; high expression is relatively more frequent in AITL, ALK-negative ALCL, and T-LBL (solid arrow), while low expression is relatively more frequent in ALK-positive ALCL and NKTL (dotted arrow). The case number in the "others" category is too small (only 4 cases), and therefore, the NOVA1 expression may not be representative of this category. PTCL, peripheral T-cell lymphoma; PTCL-NOS, peripheral T-cell lymphoma, not otherwise specified.

\section{DISCUSSION}

In this study, we found that NOVA1 was frequently and highly expressed in various subtypes of T/NK-cell lymphomas, with about $60 \%$ of T/NK-cell lymphoma cases showing high NOVA1 expression, whereas only a small number of $\mathrm{T}$ cells expressed NOVA1 in the hyperplastic palatine tonsils. As for B-cell lym- phomas, NOVA1 was negative in various types of B-cell lymphomas other than DLBCL, or normal B cells. In DLBCL, NOVA1 was expressed in a few cases; however, high expression was not noted in any cases.

Our previous study ${ }^{6}$ revealed high expression of NOVA1 in activated $\mathrm{T}$ cells and the present study also confirmed that finding. Moreover, NOVA1 was upregulated in lymphomas derived 


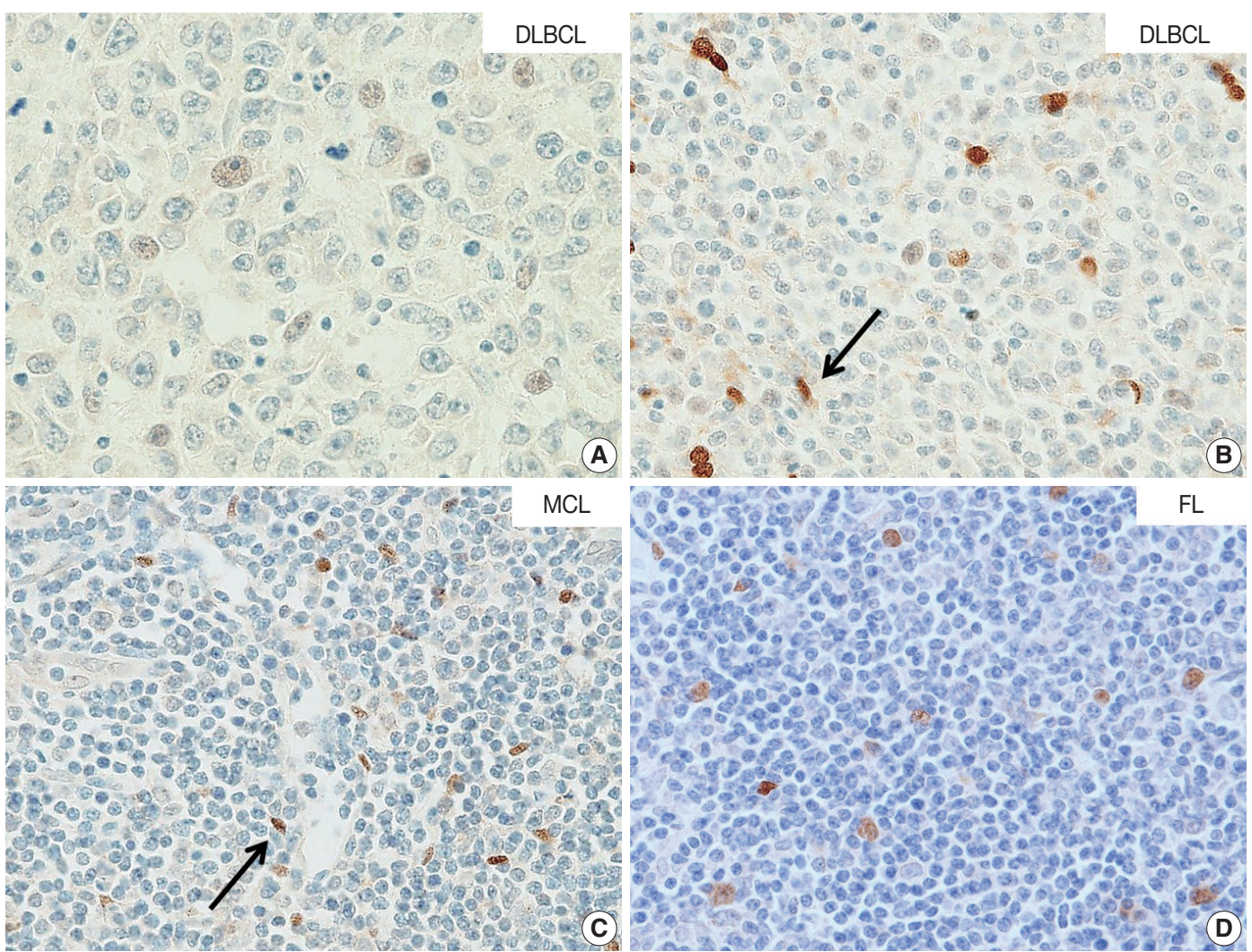

Fig. 3. Neuro-oncological ventral antigen 1 (NOVA1) expression in B-cell lymphomas. (A, B) In the representative cases of diffuse large B-cell lymphoma (DLBCL), almost all tumor cells are negative for NOVA1. In some DLBCL cases, NOVA1-expressing fibroblasts/stromal support cells are occasionally found throughout the microenvironment (arrow in panel B). (C, D) In cases of mantle cell lymphoma (MCL) or follicular lymphoma (FL), tumor cells are negative for NOVA1, and fibroblasts/stromal support cells are positive for NOVA1 (arrow).

from immature $\mathrm{T}$ cells such as precursor T-LBL and NKTL. However, in contrast to T/NK cells, NOVA1 might not be involved in B cell lesions, either malignant lymphomas or non-neoplastic $\mathrm{B}$ cells, as shown in our studies.

Among subtypes of T/NK-cell lymphomas, the expression level in tumor cells was different. Regarding the different biological characteristics and pathogenesis of each subtype of T/NKcell lymphoma, NOVA1 expression may be affected by various intrinsic biological factors related to each subtype. The AITL, ALK-negative ALCL, and T-LBL subtypes, which showed distinctively high NOVA1 expression, are biologically characteristic entities. ${ }^{11}$ Interestingly, the NOVA1 expression pattern was different even within ALCL specimens, according to ALK expression status. ALK-negative ALCL showed distinctively high NOVA1 expression while ALK-positive ALCL exhibited low NOVA1 expression. Both types of ALCL share common morphologic features of $\mathrm{CD} 30$ positivity, large anaplastic "hallmark" cells, frequent loss of pan-T-cell antigens, and expression of cytotoxic molecules, and are classified as the same disease. However, ALK-positive ALCL and ALK-negative ALCL are thought to be

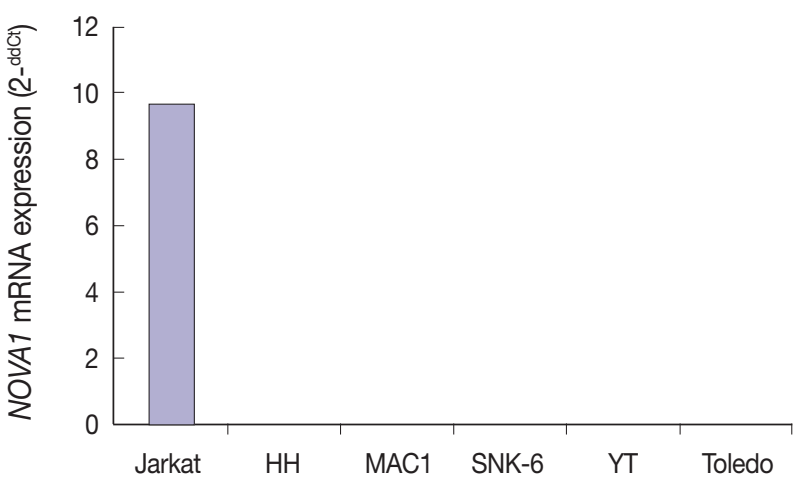

Fig. 4. Neuro-oncological ventral antigen 1 (NOVA1) mRNA expression in various cell lines. NOVA1 mRNA are expressed only in Jurkat cell lines, which correspond to T lymphoblastic leukemia/ lymphoma. NOVA1 mRNA are not detected in other tested cell lines of $\mathrm{HH}, \mathrm{MAC1}$, SNK6, YT, or Toledo.

different entities; the clinical features, prognosis, and gene expression signatures have been noted to be different. ${ }^{11}$ Therefore, the differences in NOVA1 expression may be related to the intrinsic biologic characteristics of these two types. There are no known studies that can explain the distinctively low NOVA1 

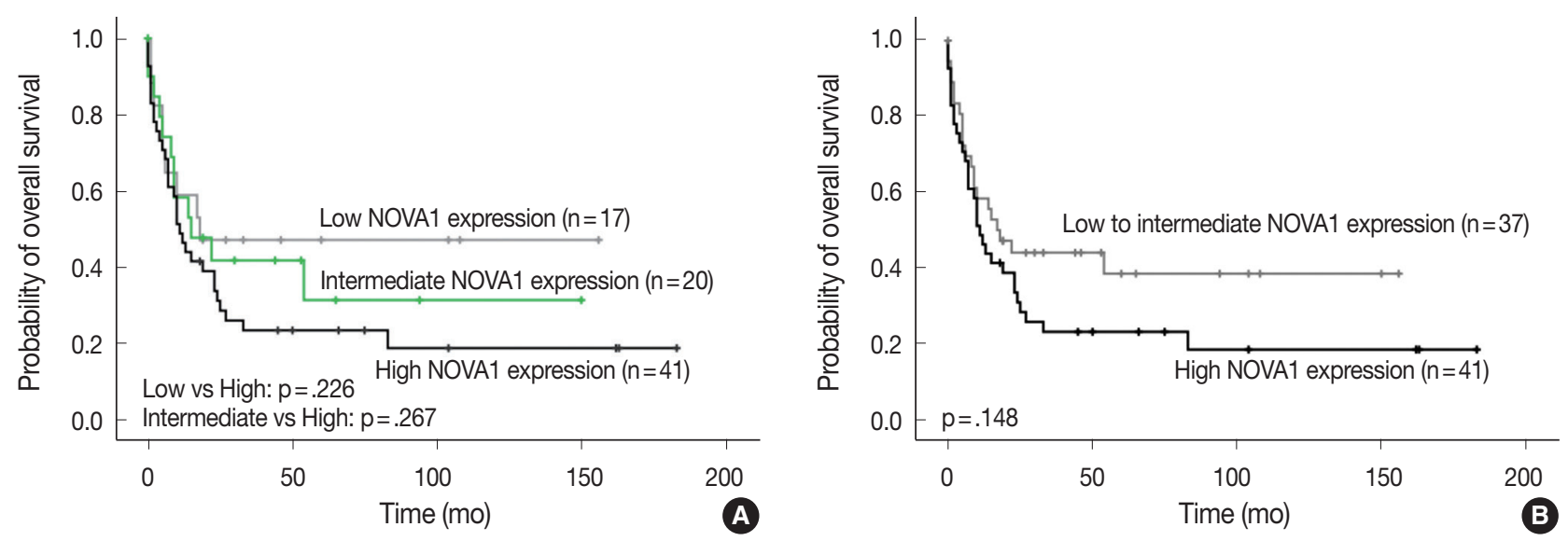

Fig. 5. Patient overall survival according to neuro-oncological ventral antigen 1 (NOVA1) expression in peripheral T-cell lymphoma, not otherwise specified; three-tiered (A) and two-tiered (B) analysis by Kaplan-Meier with log-rank test. High expression of NOVA1 tends to be related to shorter overall survival than low or intermediate expression, although statistical significance is not observed.

expression in ALK-positive ALCL. We thought that nucleophosmin (NPM), the fusion partner of ALK-NPM translocation/ $\mathrm{t}(2 ; 5)$ in ALK-positive ALCLs, may be associated. NPM is a putative ribosome assembly factor involved in processing preRNA molecules. ${ }^{13}$ Mutated NPM molecules are abnormally localized in the cytoplasm because of impaired nuclear localization, and this abnormal localization of NPM is thought to be associated with abnormal ribosome biogenesis. ${ }^{13}$ The aberrant function of mutated NPM may be related to inhibiting NOVA1 function in the splicing process of pre-RNA molecules via abnormal ribosome biogenesis. PTCL-NOS showed a relatively diverse pattern of NOVA1 expression. The proportion of cases showing an intermediate level of NOVA1 expression was relatively higher than that in other subtypes. This difference may be because PTCL-NOS is a disease group of heterogeneous entities of mature (peripheral) T-cell lymphomas.

NOVA1 expression did not vary according to the organ site where the lymphoma developed. These findings indicate that NOVA1 expression may be primarily dependent on the intrinsic characteristics of the tumor cell itself rather than extrinsic factors such as the organ-specific microenvironment, which is pre-formed before tumor growth. In association with patient prognosis, NOVA1 expression showed no statistical significance. However, in mature (peripheral) T-cell lymphomas, high NOVA1 expression tended to be related to an inferior overall survival rate. In our previous study, high NOVA1 expression levels in reactive, non-neoplastic (mature) $\mathrm{T}$ cells, which accumulate within the tertiary lymphoid structures of gastric carcinoma microenvironment, were associated with favorable patient prognosis. Suppression of NOVA1 in such reactive T cells was related with poor patient prognosis, and this effect is thought to be related to the suppression of regulatory T cell functions. ${ }^{6}$ From these findings, the role of NOVA1 in tumor T cells of malignant lymphomas and non-tumor $\mathrm{T}$ cells of tissues seems to be different. In normal physiologic conditions, NOVA1 upregulation seems to be involved in normal immune regulation. In neoplastic T cells, however, NOVA1 upregulation seems to be involved in tumor progression.

Alternative splicing of pre-mRNA is the main mechanism of transcriptome and proteome diversity, ${ }^{4,14}$ and the splicing program relies on the action of splicing factors ${ }^{1}$ like NOVA1. The action of splicing factors is cell type-specific and independent of organ origin. ${ }^{2,3,5}$ Previous studies have suggested or shown that normal and tumorous hematopoietic cells are regulated by specific alternative splicing programs and alternative transcript variants such as c-myb, a transcription factor of hematopoietic cells; CD 45, a transmembrane protein tyrosine phosphatase of T cells, $\mathrm{B}$ cells, and other hematopoietic cells; and CD20, a surface marker of mature B cells. ${ }^{15-17}$ Likewise, NOVA1 may work as an alternative splicing factor in the lymphoreticular system and in lymphomagenesis of $\mathrm{T} / \mathrm{NK}$-cell lineage.

Among the tested cell lines, only Jurkat cells, which corresponded to immature T cells, expressed the NOVA1 gene. These findings could partially support the findings observed in clinical patient samples. The functional role of NOVA1 in lymphoid cells and target genes regulated by NOVA1 in lymphomagenesis should be identified through further studies.

In summary, NOVA1 was frequently upregulated in various subtypes of T/NK-cell lymphomas. NOVA1 expression may be a new biomarker for T/NK-cell lymphomas and should be studied as a possible therapeutic target for this type of lymphoma. 


\section{Conflicts of Interest}

No potential conflict of interest relevant to this article was reported.

\section{Acknowledgments}

The study was supported by the Basic Science Research Program through the National Research Foundation of Korea (NRF) funded by the Ministry of Education, Science and Technology (2012R1A1A2007344).

\section{REFERENCES}

1. Barash Y, Calarco JA, Gao W, et al. Deciphering the splicing code. Nature 2010; 465: 53-9.

2. de la Grange P, Gratadou L, Delord M, Dutertre M, Auboeuf D. Splicing factor and exon profiling across human tissues. Nucleic Acids Res 2010; 38: 2825-38.

3. Merkin J, Russell C, Chen P, Burge CB. Evolutionary dynamics of gene and isoform regulation in mammalian tissues. Science 2012; 338: 1593-9.

4. Kalsotra A, Cooper TA. Functional consequences of developmentally regulated alternative splicing. Nat Rev Genet 2011; 12: 715-29.

5. Mallinjoud P, Villemin JP, Mortada H, et al. Endothelial, epithelial, and fibroblast cells exhibit specific splicing programs independently of their tissue of origin. Genome Res 2014; 24: 511-21.

6. Yoon SO, Kim EK, Lee M, et al. NOVA1 inhibition by miR-146b-5p in the remnant tissue microenvironment defines occult residual disease after gastric cancer removal. Oncotarget 2016; 7: 2475-95.

7. Drayton DL, Liao S, Mounzer RH, Ruddle NH. Lymphoid organ development: from ontogeny to neogenesis. Nat Immunol 2006; 7: 344-53.
8. Ruddle NH, Akirav EM. Secondary lymphoid organs: responding to genetic and environmental cues in ontogeny and the immune response. J Immunol 2009; 183: 2205-12.

9. Buckley CD, Barone F, Nayar S, Bénézech C, Caamaño J. Stromal cells in chronic inflammation and tertiary lymphoid organ formation. Annu Rev Immunol 2015; 33: 715-45.

10. Kalluri R, Zeisberg M. Fibroblasts in cancer. Nat Rev Cancer 2006; 6: 392-401.

11. Swerdlow SH, Campo E, Harris NL, et al. WHO classification of tumours of haematopoietic and lymphoid tissues. $4 \mathrm{~h}$ ed. Lyon: IARC Press, 2008.

12. Park E, Park SY, Kim H, et al. Membranous insulin-like growth factor-1 receptor (IGF1R) expression is predictive of poor prognosis in patients with epidermal growth factor receptor (EGFR)-mutant lung adenocarcinoma. J Pathol Transl Med 2015; 49: 382-8.

13. Falini B, Nicoletti I, Bolli N, et al. Translocations and mutations involving the nucleophosmin (NPM1) gene in lymphomas and leukemias. Haematologica 2007; 92: 519-32.

14. Kelemen $\mathrm{O}$, Convertini $\mathrm{P}$, Zhang $\mathrm{Z}$, et al. Function of alternative splicing. Gene 2013; 514: 1-30.

15. Oberdoerffer S, Moita LF, Neems D, Freitas RP, Hacohen N, Rao A. Regulation of CD45 alternative splicing by heterogeneous ribonucleoprotein, hnRNPLL. Science 2008; 321: 686-91.

16. O'Rourke JP, Ness SA. Alternative RNA splicing produces multiple forms of c-Myb with unique transcriptional activities. Mol Cell Biol 2008; 28: 2091-101.

17. Henry C, Deschamps M, Rohrlich PS, et al. Identification of an alternative CD20 transcript variant in B-cell malignancies coding for a novel protein associated to rituximab resistance. Blood 2010; 115: $2420-9$. 
Appendix 1. Clinicopathological characteristics of T/NK-cell lymphoma cases

\begin{tabular}{|c|c|}
\hline Characteristic & No. $(\%)(n=139)$ \\
\hline Sex (male vs female) & $94(67.1): 45(32.9)$ \\
\hline Age $(<60$ yr vs $\geq 60 \mathrm{yr})$ & $91(65.0): 49(35.0)$ \\
\hline \multicolumn{2}{|l|}{ Subtype } \\
\hline NKTL & $45(32.4)$ \\
\hline PTCL & $38(27.3)$ \\
\hline AITL & $14(10.1)$ \\
\hline ALCL, ALK-negative & $12(8.6)$ \\
\hline ALCL, ALK-positive & $12(8.6)$ \\
\hline T-LBL & $15(10.8)$ \\
\hline Others & $3(2.2)$ \\
\hline \multicolumn{2}{|l|}{ Primary site of tumor } \\
\hline H\&N vs LN vs GI vs soft tissue and bone vs others (solid organs) & $37(26.4): 72(51.4): 9(6.4): 14(10.0): 8$ (5.7) \\
\hline Ann Arbor stage (I \& II vs III\&IV vs not evaluable) & $29(27.9): 77(55.0): 34(24.3)$ \\
\hline LDH level (normal vs elevated vs not evaluable) & $39(27.9): 60(42.9): 41(29.3)$ \\
\hline BM involvement (absent vs present vs not evaluable) & $77(55.0): 33(23.6): 30(21.4)$ \\
\hline IPI score (0-2 vs 3-5 vs not evaluable) & $65(46.4): 43(30.7): 32(22.9)$ \\
\hline \multicolumn{2}{|l|}{ Initial treatment modality } \\
\hline CTx alone vs CTx with RTx vs RTx alone vs no Tx vs not evaluable & $63(45.0): 27(19.3): 1(0.7): 1(0.7): 48(34.3)$ \\
\hline
\end{tabular}

Appendix 2. List of primary antibodies and their dilutions

\begin{tabular}{llll}
\hline Antibody & Dilution & Type & Company \\
\hline CD20 & $1: 1600$ & Mouse & DAKO, Glostrup, Denmark \\
CD3 & $1: 200$ & Rabbit & LabVision, Fremont, CA, USA \\
CD4 & $1: 200$ & Rabbit & Cell Marque, Rocklin, CA, USA \\
CD8 & $1: 100$ & Mouse & Cell Marque, Rocklin, CA, USA \\
CD56 & $1: 200$ & Rabbit & Cell Marque, Rocklin, CA, USA \\
\hline
\end{tabular}

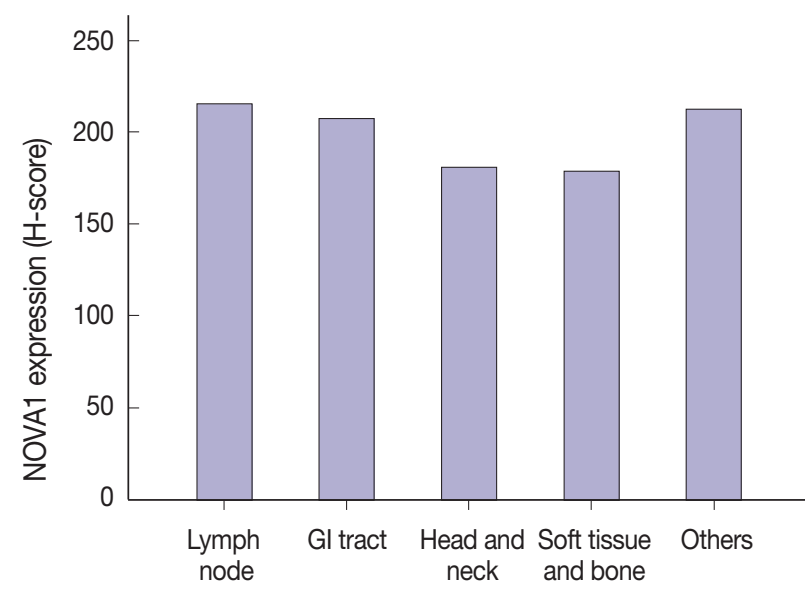

Appendix 3. Neuro-oncological ventral antigen 1 (NOVA1). NOVA1 protein expression in various organ/sites of lymphomas. NOVA1 expression level in tumor cells does not vary according to the organ/sites where lymphomas were growing. Gl, gastrointestinal. 